\title{
WHAT MIGHT BE RIGHT ABOUT THE CAUSAL THEORY OF TIME
}

A causal theory of time, or, more properly, a causal theory of spacetime topology, might merely be the claim that according to some scientific theory (the true theory? our best confirmed theory to date?) some causal relationship among events is, as a matter of law or merely as a matter of physically contingent fact, coextensive with some relationship defined by the concepts of the topology of the spacetime. A strongest version of such a causal theory would be one which demonstrated such a coextensiveness between some causally definable notion and some concept of topology (such as 'open set') sufficient to fully define all other topological notions. Given such a result, one would have demonstrated that for each topological aspect of the spacetime, a causal relationship among events could be found such that that causal relationship held when and only when the appropriate topological relationship held.

I believe, however, that the aim of those philosophers who have espoused causal theories of spacetime topology has been grander than this. There is a general and familiar philosophical program that works like this: In some given area of discourse it is alleged that our total epistemic access to the features of the world described in this discourse is exhausted by access to a set of entities, properties and relations characterized by an apparently proper subset of this conceptual scheme. Then, it is alleged, the full content of propositions framed in this discourse must be characterizable in terms of its totality of observational content, this content being describable in the distinguished concepts of the subset of epistemically basic concepts. So, the program continues, it is up to us to show how all the empirical consequences of the theory really are framable in the distinguished concepts alone. In so far as the theories framed in the totality of concepts appear to make empirical claims outrunning those expressible in the basic concepts, these must be shown to be ultimately 
reducible to the empirical content expressible in the basic concepts. And if the assertions of the total theory go beyond those reducible in content to assertions of the distinguished vocabulary, then they must be shown to be true merely as a matter of choice or 'convention'.

I believe that, explicitly or implicitly, some such program underlies all those various causal theories of spacetime topology with which we are familiar. So interpreted the philosophical theory is not just the explication of the results of some scientific theorizing, but an attempt at an epistemological and semantic critique which displays initial constraints into which any satisfactory scientific theory must fit. For if the epistemic and semantic analysis proposed by the philosophical theory is correct, then the limits of meaningful assertiveness of the scientific theories are delimited, the distinction between fact asserting and 'mere convention making' elements in the scientific theories is drawn, and the criteria are made clear under which we are entitled to say whether or not two allegedly alternative total theories are or are not properly speaking 'equivalent' to one another and, in Reichenbach's terms, whether they differ merely in descriptive simplicity or in some genuine empirical content.

Taking our total theory to be one which describes the spatiotemporal topological structure on events and that portion of the causal relationships among them which contains reference only to which events are and are not causally connectible with one another, a causal theory of spacetime topology is a philosophical theory which alleges that the total empirical content of this overall theory is exhausted by its causal part; and that any spatiotemporal topological assertion can be reduced to an assertion about causal relatability supplemented, perhaps, by conventional choices of 'ways of speaking'.

But, I believe, we must proceed with some caution here. What counts as 'the full causal structure on events', and, hence, what counts as the allowable empirical basis to which all our theoretical elements are to be reduced may not be the same in all 'causal' theories of spacetime topology. And two philosophical theories even while agreeing as to the nature of the 'epistemic basis', may, as we shall see, disagree as to why the elements in question are allowed into the reduction base.

What I wish to suggest here is that, for rather well known reasons, some choices of an epistemological basis in causal theories of spacetime 
topology won't do the job intended for them. I think that one suggested basis does have some plausibility. But its plausibility, I will suggest, rests on grounds which might lead us to think that the designation 'causal' is inappropriate for this philosophical reductionist account of spacetime topology.

While our ultimate grounds for accepting or rejecting such a 'philosophical' theory of spacetime topology will be 'philosophical' or, more particularly, in terms of an epistemological critique of spacetime theories, it is important and enlightening to see just how particular scientific theories of spacetime fare in the light of one's philosophical account. I will try to show how several recent results in the mathematical foundations of general relativity 'fit in' with the analysis of implausible and more plausible 'causal' theories of topology which I will survey.

Finally, while I believe that the 'causal' theory I end up with is a more plausible candidate for a possibly successful reductionist account of spacetime topology, I do not believe that it itself is uncontrovertibly correct. I will note some reasons for being cautious about accepting it as a philosophical account of spacetime epistemology and semantics.

Let us consider first the version of the theory which takes as its 'reduction basis' the relation of causal connectibility among events. As usual, we note, the 'definitions' of the topological notions in terms of the causal are in the mode of possibility. Some topological relationship is alleged to hold if some appropriate causal relationship could hold. That topological notions reduce to causal only if we allow causal notions in the mode of possibility' is a familiar feature of such philosophical reductionism. I will forego rehearsing the well-known reasons for the necessity of the invocation of such possibility talk and the equally familiar reasons for alleging that such an invocation vitiates at least part of the reductionist program.

In the version we are now exploring the notion of two events being causally connectible, i.e. its being possible for one to be causally related to the other, is taken as our primitive notion. In terms of it we attempt to construct an adequate defining basis for the full gamut of spatiotemporal topological notions. Not surprisingly we do not need to invoke any notion of the direction of time at this point, and causal connectibility, rather than 
the asymmetric relationship of ' $a$ could be a cause of $b$ ' will be sufficient. ${ }^{\text {' }}$ How plausible is such a causal theory of topology?

There are, I believe, two basic grounds on which a philosophical reductionist thesis is usually challenged:

(1) First it might be claimed that in order to have grounds for believing that a relationship utilized in the reduction basis holds, we must first have some knowledge of relationships holding which are in the portion of the theory to be reduced or defined away. If this is so, it is claimed, then the reduction basis is not a properly epistemically primitive basis.

(2) Second it can be argued that the reduction basis is not adequate to provide 'definitions' for all the concepts of the total theory. That is, even if we can, without knowing any of the defined relationships to hold, establish which relationships in the reduction basis do in fact hold, that information will not be adequate to fully determine all the relationships which are expressed in the total theory.

Our first version of a causal theory of spacetime topology has been challenged on both these grounds.

First, can we really tell which events are causally connectible without already knowing a great deal about their spatiotemporal relationships to one another? In our usual accounts of the notion of causality we find that while causality means falling under a natural law, and a law is a general rule connecting kinds of events, knowing which particular events are causally related to which other particular events requires knowing a great deal about the spatiotemporal relations of these events to one another. Strikings of matches cause lightings of matches, but to tell that this striking caused that lighting we must know that this particular striking bears to that particular lighting an appropriate spatiotemporal relationship. Now while I believe that there is some truth to this objection, I think that a possibly viable 'causal' theory of topology is lurking under this first theory despite this initial objection. But rather than pursue the matter here, I will save this discussion for later. ${ }^{2}$

Second, can we really define all our topological notions in terms of the notion of causal connectibility? Here is where some results of the mathematical study of spacetimes become crucial. If the philosophical reductionist theory is correct, it is claimed, then in any possible spacetime we envision the topological notions must be connected to the appropriate causal notions in the way specified by the reductionist account. For the 
topological notions are supposed to be defined by the causal notions. Is there a definition of the appropriate basis for all topological notions (say a definition of 'open set') in terms of causal connectibility which holds in all the spacetimes we take as conceivable?

Not if we allow ourselves the full range of spacetime compatible with general relativity, including those we might view as causally 'pathological'.

In the Minkowski spacetime of special relativity we can indeed causally define (in the present meaning of that term) the open set basis sufficient to fully define the topology. An explicit definition of the open sets in terms of causal connectibility is available in terms of the well-known Alexandroff topology for Minkowski spacetime.

In those general relativistic spacetimes which are strongly-causal it can also be shown that the Alexandroff topology and the usual manifold topology will coincide. Indeed, the coincidence of the topology defined by the causally defined open sets of the Alexandroff topology with the usual manifold topology is equivalent to strong causality. When the spacetime is not strongly-causal, however, the manifold topology and the Alexandroff topology will fail to coincide. ${ }^{3}$

Malament has recently shown that in any spacetime which is both past and future distinguishing (a weaker condition than strong causality) one can at least implicitly define the topology in terms of causal connectibility. That is, given any two past and future distinguishing spacetimes, any causal isomorphism between them (bijection preserving causal connectibility) will be a homeomorphism relative to the usual manifold topology. He has also shown that this is a strongest possible result in that one can construct examples of spacetime in which either past or future distinguishing is violated and such that there will be causal isomorphisms between pairs of such spacetimes which fail to be homeomorphisms. If the past and future distinguishing condition is not met the topology cannot be even implicitly defined in terms of causal connectibility. There are, in fact, spacetimes where the failure of causal definition of the topology is made particularly manifest. I refer here to those spacetimes where the topology is non-trivial but where causal connectibility is a relationship which holds between every pair of events. ${ }^{4}$

Now we might try to hold that the kinds of spacetimes in which either explicit or implicit definition of the topology by causal connectibility fails 
are impossible spacetimes. For example, they are, to be sure, infected with the kind of pathological causality which we would not ordinarily expect to find in a causally 'well behaved' universe. But if we can even understand what such spacetimes would be like, then the fact that we can understand them (know what it would be like to live in one of them, for example) shows us, I think, that any hopes of establishing that in the actual world our actual concepts of topology are defined by notions of causal connectibility are unacceptable. Without going into details here, I believe that such worlds are perfectly intelligible to us and that with a little imagination anyone familiar with the basic concepts of relativistic spacetime can be gotten to understand just what the topological aspects of such a world would be like to an inhabitant of them. Further, we can construct pairs of spacetimes which are causally isomorphic in our present sense and which are such that an inhabitant of them can, by topological exploration, discover in which of the alternative spacetimes he resides. ${ }^{5}$

Now the fact that our initial attempt at a causal theory of spacetime topology fails in two diametrically opposite directions might lead us to believe that the prospects for any such theory are dismal indeed. The basis chosen in this original version of the theory is too weak to do the job required of it - define the full topological structure in every spacetime we wish to take as intelligible. Yet it is so strong, i.e. contains so many elements and of such a kind, that it appears that we already need epistemic access to much spatiotemporal structure on events in order to tell when the relationship which is utilized in the basis, causal connectibility, holds. But we will see that the situation is not quite as hopeless as it looks.

Let us try to remedy the defects of our original theory first by moving to a stronger basis, holding in abeyance for the moment the obvious difficulties this will give rise to. Within the relativistic context two events are causally connectible if there is a continuous causal (timelike or lightlike) path between them. This follows from the standard view that causal interaction is inevitably mediated by the emission, transmission and reception of some 'genidentical' material or lightlike 'particle'. So within this context, we know that the relationship of causal connectibility 
holds of a pair of events if we know the truth of the existential claim that there is at least one continuous causal path containing the two events.

Now suppose we take as our basis notion not causal connectibility but, instead, the notion of continuous causal path. We then have full knowledge of the basis relationship not when we know merely which pairs of events are connectible by some continuous causal path or other, but when we can tell of any set of events whether or not it constitutes a continuous causal path. Does the introduction of this much stronger basis change the picture significantly?

That is does follows from an important and interesting result of Malament's. Given any two spacetimes (taken to be four-dimensional manifolds with pseudo-Riemannian metrics of Lorentzian signature) any bijection which takes continuous causal curves into continuous causal curves will be a homeomorphism! In other words, the full topology of the spacetime will be fixed by its class of continuous causal curves. So if we can fully determine the latter, then we can fully specify the former as well. ${ }^{6}$

But can we determine the continuous causal curves of a spacetime without already being able to determine its topological features? This obvious objection to the epistemological use of a result like Malament's to establish a causal theory of topology is well known. Let us first present it and then in the next section move on to the deferred task of showing why, despite this argument, something which might be called a causal theory of topology can have a case made out for it that meets this 'epistemological' objection.

In one version, and among philosophers I think the most common one, the picture one has is something like this: A single observer wishes to map out the topology of the spacetime in which he dwells. Equipping himself with an infinite number of material or lightlike particles, he emits them from all points in all directions (we must be generous in such idealizations) so determining the structure of continuous causal paths in his spacetime. Now, by Malament's result, he can pin down uniquely the full topology of the spacetime, including such not-directly-determined features as which spacelike paths are continuous, etc.

But how does our observer tell which classes of events in the life history of a 'genidentical' particle are spatiotemporally continuous portions of its history? Indeed, how does he even tell which events are events in the 
history of one such particle and not events selected at random from the histories of any number of distinct particles? Only, it is alleged, by already knowing what the continuous spatiotemporal segments really are, i.e. only by already having access to topological features of the spacetime. ${ }^{7}$

I V

But consider the following version of a 'causal' theory of topology: It is true that on the picture just looked at we gain access to the full topology of the spacetime only by already knowing part of it, i.e. only by already knowing which are the continuous timelike and lightlike curves in it. But this is already a 'reductionist' gain. And when we realize the real epistemological importance of the basis to which total topological knowledge has been reduced, we see just how important a gain this is. For what constitutes the continuous causal paths of the spacetime is just what is available to our direct epistemic access. Not because we have some wonderful way of spotting continuous segments in the history of genidentical particles without any antecedent knowledge of the topology. That is silly, for what we mean by continuous segment in the history of a genidentical particle is just a continuous set of spacetime locations along a causal path occupied by the same kind of material particle. But, rather, because each causal path can be traversed by a local 'consciousness' who directly and immediately, as a primitive content of his experience, can tell which events in his consciousness, and hence which spacetime locations along the worldline of his history, are 'near' one another!

The idealized picture we have now is of a universe equipped not with one observing 'consciousness' but with a plentitude of them, so that the totality of causal worldlines of the spacetime is covered by consciousnesses sensing immediately and directly which locations of the spacetime along their respective worldlines are 'near', i.e. determining without inference or instrument which are the continuous segments of causal curves in the spacetime. Extravagant as this seems when put this way, the basic idea here has appeared explicitly or implicitly from Robb through Hawking and Malament. ${ }^{8}$

So the causal theory of spacetime topology has now been replaced by a reductionist account which is not really 'causal' in its fundamental epistemic motivation. The reductionist program still takes as the body of 
concepts to be reduced those characterizing the full topology of the spacetime. The reduction basis consists in those concepts relating to continuity of the one-dimensional causal worldlines of the spacetime. But this set of topological features is not discriminated from the general class because the causal worldlines are the paths of propagation of causal influence throughout the spacetime but, rather, because such curves, being the worldlines of the possible life histories of 'consciousnesses', have their continuity open to 'direct epistemic access' without the intervention of instruments or the necessity of theoretical inference. I will shortly return to the question of just how plausible such a reductionist program might be. I don't wish here to maintain that this is the correct philosophical account of our knowledge of the topology of spacetime and of the semantic analysis of topological concepts in general, but only that it is the most plausible version of a 'causal' theory of topology, combining, as it does, a genuine reduction (from the totality of topological aspects to those along causal paths alone) with an at least prima facie case for the epistemic priority (in terms of immediacy of access) of the concepts of the reduction basis. I also believe that at least some readers will agree with me that it is this version of the 'causal' theories which captures the fundamental epistemic 'intuitions' which lay behind the other more 'causal' causal accounts.

At this point it is enlightening to introduce some additional results of recent mathematical work on spacetimes. At first glance these new results might seem to militate against the version of the causal theory we ended up with. But on reflection, I believe, they illustrate, rather, the force of our last account of the causal theory.

Suppose we have available to us a full picture of the topology (continuity) of the causal worldlines of the spacetime. Can we then infer immediately the full topology of the spacetime? Malament's result seems to show that we can, for any two spacetimes (in the usual 4-manifold with Lorentz-signature metric sense) which agree on their causal worldline topology will agree in all topological aspects. But it isn't that simple.

Following the work of Zeeman on novel topologies for Minkowski spacetime, recent work has been done on looking for interesting novel topologies for the spacetime of general relativistic worlds. These new 
topologies, unlike the usual manifold topology, 'code' into themselves the full causal structure of the spacetime. This is true in the sense that any homeomorphism between spacetimes relative to the new topologies are automatically conformal isometries. This is not true of homeomorphism relative to the usual manjfold topologies. With these new topologies the spacetimes are not manifolds. ${ }^{9}$

The importance of these new topologies for our present purposes can be seen in the manner of their construction. One starts off imagining a spacetime equipped with the usual manifold topology. One then seeks a new topology which will code the causal structure in the manner described above, and which will then, perforce, differ from the usual manifold topology. But the new topology is so constructed that it agrees with the manifold topology on the topology induced on the one-dimensional causal curves. For example, there is the path topology of Hawking, King and McCarthy. It is defined as being the finest topology on the spacetime which induces on all continuous causal curves the same topology induced on them by the usual manifold topology.

So, obviously, the standard and the novel topologies will agree on all topological facts about the causal curves. Now aren't we in a position to be skeptical about our 'causal theory of topology'? For if we can fully exhaust our knowledge about the topology along such causal paths and yet still not know the full topology of the spacetime (is it the standard manifold topology or is it, instead, one of the novel topologies?) aren't we in just the same position which caused us to reject our first version of a causal theory of topology? For there we pointed out that in the case of spacetimes which were not both past and future distinguishing, two manifolds could share their causal structure (there could be a causal isomorphism between them) and yet not be topologically identical (i.e. not homeomorphic). This led us to say that the structure determined by causal connectibility was not sufficient, in general, to fully fix the topology of the spacetime. And here, once again, we seem to be saying that once we allow for the possibility of the novel topologies, we can no longer fully fix the topology of the spacetime even given full topological knowledge of the causal paths.

But the situations are not parallel. The trouble with the causal connectibility version of the causal theory of topology is this: Two spacetimes might be causally isomorphic and yet be empirically distinguishable in 
their topological structure. They may very well differ in the structure of continuity along causal paths which is the paradigm of epistemically accessible topological structure. But if two spacetimes share the same topological structure along the causal paths, and if this structure exhausts the empirically determinable topological structure of spacetime, then no empirical observation could tell us which of two incompatible full topologies (say standard vs. the path topology of Hawking et al.) is really the full topology.

And what that suggests is this (the familiar end product of the problem of theoretical conventionality): If two spacetimes share the same topology induced on the causal paths, then, appearances to the contrary, they are really (topologically) the same spacetime. Their real topological structure may be expressed in the standard manifold form (and, as Malament's theorem shows only one of these will be compatible with the topology on the causal paths) or it may be expressed in terms of the path topology of Hawking et al., or in terms of any other non-manifold topology designed to code the causal structure. But these are merely alternative formulations of one and the same set of empirical facts. For (in the usual positivist vein) since the topology on the paths exhausts what can be empirically discovered about the topology in general, the total factual content of the general topology is exhausted by the topological structure it induces on the causal paths.

One awaits at the present time a neat mathematical formulation which eschews the necessity for topological assertions about the spacetime which appear to outrun the topological facts about causal curves, and which captures the empirical equivalence among all topological structures which induce the same topology on the causal paths, by offering as the full characterization of the topology of the spacetime the mathematical description of the continuity structure on causal paths alone.

VI

How plausible is a causal theory of spacetime topology, even in its most plausible version in which it becomes rather a 'topology of worldlines traversable by a consciousness' theory of spacetime topology? Now, of course, much of the answer to that question will depend upon just how plausible in general one takes philosophical reductionist theses, with their 
underlying positivist motivations, to be. While many are skeptical (often without much in the way of argument above and beyond 'realist' dogmatism) of reductionism in general, this is hardly the place to rehearse most of the familiar issues of whether or not 'pure' observation bases exist, of the allowability of modal possibility talk into the reductive definitions, etc. Rather let me focus on just a few issues for discussion which come to mind when one reflects on this particular attempt at philosophical reductionism. ${ }^{10}$

(1) The theory presupposes that at least one aspect of spacetime structure is accessible to consciousness without instrument or inference. But is this belief supportable? Here the reader may remember the discussion of spatiotemporal coincidence in Reichenbach's Axiomatization of the Theory of Relativity. Here Reichenbach reflects upon the fact that while coincidence is taken as a primitive of his (and everyone else's) reconstruction of relativity, if this decision to take it as a primitive is based upon the ground that we have immediate non-inferential knowledge of what events in the physical spatiotemporal world are really coincident we are on dangerous ground. Aren't the coincidences of which we are 'immediately' aware subjective coincidences; and isn't it objective coincidence with which we are concerned in our reconstruction of the theory of physical spacetime? ${ }^{11}$

In the present case, by what right do we identify the continuity of inner experience of some observer, as subjectively experienced, with the 'real' physical continuity of the spacetime locations at which these experiences occur? Imagine, for example, a consciousness instantaneously and discontinuously transported through spacetime, so that the continuity of his 'inner' experiences misrepresents the discontinuity of the spatiotemporal locations at which he has them.

What we have here is just one more example of a well known and enormous difficulty for reductionism: If reductionism allows in its basis for reconstruction only the content of the immediately experienced, then how can it ever give us an adequate account of the nature of the objective, physical world? For if we slide down the slippery slope we get on to when we once begin to 'reduce' the inferred to the 'immediately experienced', how can we ever stop before reaching the bottom where the basis is the contents of subjective experience; thereby dropping out of the realm of concepts dealing with the physical 'outer' world altogether? 
(2) The theory presupposes that our only direct epistemic access is to the continuity of spacetime locations in the lived one-dimensional history of a consciousness traversing a causal worldline. Is this plausible?

First, relying on something quite reminiscent of Kant's distinction between space as the manifold of apperception of outer experience and time as the manifold of apperception of both outer and inner experience, the theory places our experience of time, and continuity in it, in a very special position indeed. All our topological insight into the world is to be grounded in an awareness only of the temporal continuity of our experience. Our knowledge of the spatial aspects of the world is derivative from this temporal sense, and, if what we have said about conventionally alternative topologies is correct, really, properly speaking, only conventional. For the only real topological facts are the facts about temporal continuity along causal paths.

But is this correct? What about our apparent 'direct' knowledge of the structure of the space around us? Is this to be dismissed? We could, of course, pull the not uncommon move of distinguishing 'perceptual space' from 'physical space' and argue that our direct apprehensions are only of the former, the latter to be 'constructed' out of our temporal experience. But then the peculiar asymmetry of taking most of our perceptual life to be 'merely subjective' while allowing our direct experience of temporal continuity to serve as immediate access to the external world appears in an even more striking light.

Consider, again, what is, on reffection, the rather surprisingly different treatment accorded topological and metrical aspects of spacetime on this view. Nearly everyone who writes about the foundations of our knowledge of spacetime takes metric features of the world, even those along causal paths (elapsed proper time along a causal worldline) to be founded on some physical measuring process. In order to ground our knowledge of just how much proper time elapses between two events on a causal path we must rely, it is almost invariably alleged, either on clocks or on a specification of the affine parameter determined by the paths of freely falling particles. If one tries to bring forward our immediate experience of the magnitude of duration, this is almost always dismissed as a confusion of 'psychological time' with the objective physical magnitude.

But why should psychological experience of temporal magnitude be irrelevant to founding our knowledge of the magnitude of physical 
duration and yet the psychological experience of temporal continuity be taken as giving us immediate access to the real facts about continuity along causal paths?

(3) Next consider Reichenbach's well known claim that the topology of spacetime is just as 'conventional' as is its metric. Just as we can save alternative metrics in the light of any experience by a sufficiently rich flexibility in the postulation of universal forces, so any topological thesis can be maintained if we allow ourselves the global flexibility of choosing how to identify or dis-identify events (moving from multiply connected spaces to their simply connected covering spaces, for example) and the local flexibility of tolerating causal anomalies, i.e. spatiotemporally discontinuous causal interaction mediated by the spatiotemporally discontinuous motion of genidentical signals. ${ }^{12}$

But if the continuity of worldlines is determinable directly by consciousness traversing them, aren't we denied at least the second kind of flexibility noted? So aren't at least the local facts about topology nonconventional? Here I think that two (obvious) options present themselves: (1) We could accept the non-conventionality of continuity along causal worldlines, thereby supporting the intuitions which at least some have had that topological facts (or at least some of them) are nonconventional in opposition to the full conventionality of the metric; or (2) We could remember the possibility, noted above, of consciousnesses themselves experiencing as continuous what are actually spatiotemporally discontinuous histories, and allow for the saving of topologies now by contemplation of a sufficiently rich allowance of 'experientially anomalous' worlds.

All of this is just one more way of emphasizing the fundamentally problematic nature of our best causal theory: the fact that it seems to put one kind of experience on our part-the experience of temporal continuity - on a pedestal as the one way in which psychological experience gives us 'direct access' to the structure of the objective world.

(4) It is worthwhile to reflect for a moment on the idealized basis of 'directly knowable facts' to which all assertions are to be reduced in our theory of topology. This consists in all facts about the continuity of causal path segments, for each such segment could be traversed by a consciousness able to directly ascertain its topology. 
But, of course, no one consciousness, even in the most extreme idealization, could traverse them all. For example, a consciousness which experiences one event is, in principle, excluded from having in its experience, ever, the direct awareness of an event at spacelike separation from this event.

Of course it is a standard problem with philosophical reductionisms of this kind as to whether the reduction basis should consist in the total possible experience of a single observer or the total amalgamated experience of all possible observers. In the relativistic context this takes on a particularly disturbing aspect. The experiences of some observers simply can't be communicated to some other observers. It is easy to cook up spacetimes such that one has a pair of them which are distinct in their topology and metric structure; whose differences are determinable on the basis of the collective experience of all possible observers; but which are such that no one observer cculd ever tell - even if he lived a worldline of infinite extent past and future - which of the two spacetimes he lived in. These are the so-called indistinguishable spacetimes. ${ }^{13}$

Should a reductionist account of topology allow in its basis of facts everything which could be known to all observers taken collectively, or should the basis consist, rather, in the possible experience of some one observer? The former alternative seems in many ways the more natural, for even on radically positivist grounds it seems unfair to exclude as 'real facts about the world' something which someone could ascertain in a direct non-inferential way. On the other hand, the usual pressures toward solipsism encountered in positivist programs may tend to make a sufficiently radical reductionist reject even the basis to which the causal theory reduces topology as allowing too much in, since it takes as factual elements of the world which could never, even in the most 'in principle' way, be known to any single observer trying to ascertain the topology of the world.

\section{VII}

Ultimately, deciding on the plausibility of some reductionist account of topology of the kind we have been exploring will require resolving some of the deepest of philosophical questions: questions relating to the existence and nature of an 'observation basis' on which all theorization is to be constructed and questions relating to just what extent one can, on 
the one hand, support a positivist-reductionist account of 'theoretical' features of the world and, on the other hand, to what extent one can defend a 'realism' which allows for valid inference 'beyond the immediately observable'. A theory of theories which does justice both to our strongly held intuitions of realism and yet at the same time makes coherent sense of the progress of physics in weeding out 'metaphysical' elements by means of epistemic critiques and Ockham's Razorish prunings of theories is not yet before us. But at least I think that we can now see that a causal theory of topology is really one more attempt at such an epistemological critique of theories.

I have been maintaining that 'causal' is really a misnomer for such theories of topology. To be sure even in our last, most plausible, version of a 'causal' theory of topology the 'hard facts' about the topology of the world are reduced to facts about the continuity and discontinuity of causal paths in the spacetime. But not because they are the paths of 'genidentical' causal signals, rather because they are the paths which constitute the possible life-histories of experiencing consciousnesses.

One final query: Is the identity of the causal paths with the worldlines of possible consciousnesses just an 'accident', just an artifact, as it were, of the relativistic facts about the lawlike nature of the world? Or is this identity within the relativistic context instead the inevitable result of any physical theory which survives an adequate epistemic critique? If the latter is really the situation, rather than the former, then the misidentification of reductionist topological theories as causal may not be merely a mistake, but rather a symptom leading us to further insight. ${ }^{14}$

The University of Michigan

NOTES

1 See R. Latzer, 'Nondirected Light Signals and the Structure of Time', Synthese 24 (1972), 236-280, and D. Malament, Ph. D. Thesis (Rockefeller University), chap. II, unpublished.

${ }^{2}$ For a statement of this kind of objection to causal theories as epistemologically motivated reductions see A. Grünbaum, Philosophical Problems of Space and Time, Knopf, New York, 1963, pp. 190-191.

${ }^{3}$ See S. Hawking and G. Ellis, The Large Scale Structure of Space-Time, Cambridge, Cambridge, 1973, pp. 196-197, and S. Hawking, A. King, and P. McCarthy, 'A New Topology for Curved Space-time Which Incorporates the Causal, Differential, and Conformal Structures', J. Math. Phys. 17 (1976), 174-181, esp. p. 176. 
${ }^{4}$ See Malament's thesis cited, chap. III and also D. Malament, 'The Class of Continuous Timelike Curves Determines the Topology of Spacetime', unpublished at the time this piece is written, p. 11, Theorem 2.

${ }^{5}$ To see this consider the example used by Malament on p. 13 of his 'The Class of Continuous...' to show that a failure of past or future distinguishingness is sufficient to generate causal isomorphisms which are not homeomorphisms.

${ }^{6}$ Malament, thesis, chap. III and 'The Class of Continuous ..., p. 8, Theorem 1.

${ }^{7}$ For this objection see H. Lacey, 'The Causal Theory of Time: A Critique of Grünbaum's Version', Philos. Sci. 35 (1968), 332-354. See also L. Sklar, Space, Time, and Spacetime, University of California, Berkeley, 1974, sec. IV, E, 3.

${ }^{8}$ See the introductory portions of A. Robb, A Theory of Time and Space, Cambridge, Cambridge, 1914 where the epistemic suitability of taking 'after' as the basic primitive for constructing a spacetime theory is discussed. See Hawking, King and McCarthy, op. cit., p. 175. Malament in 'The Class of Continuous ...', p. 1 says that this result on the implicit definability of the topology be the class of continuous causal curves is "... of interest because, in at least some sense, we directly experience whether events on our worldlines are "close' or not."

9 See E. Zeeman, 'The Topology of Minkowski Space', Topology 6 (1967), 161-170 for the introduction of these non-manifold topologies in special relativity. For the generalization to general relativistic spacetimes see Hawking, King and McCarthy, op. cit.

${ }^{10}$ For a discussion of reductionism in general and in the specific context of spacetime theories see Sklar, op. cit., sec. II, H, 4. See also L. Sklar, 'Facts, Conventions and Assumptions in the Theory of Spacetime', forthcoming in Minnesota Studies in the Philosophy of Science, vol. 8 (ed. by J. Earman, J. Stachel and C. Glymour).

${ }^{1 i}$ See H. Reichenbach, Axiomatization of the Theory of Relativity, English translation, University of California, Berkeley, 1969, pp. 16-21. See also Sklar, 'Facts, Conventions...', sec. IV, B.

${ }_{12}$ See H: Reichenbach, The Philosophy of Space and Time, Dover, New York, 1958, p. 65.

${ }^{13}$ On indistinguishable spacetimes see C. Glymour, 'Topology, Cosmology and Convention', Synthese 24 (1972), 195-218. See also Glymour's 'Indistinguishable Space-Times and The Fundamental Group' and D. Malament's 'Observationally Indistinguishable SpaceTimes' forthcoming in vol. 8 of the Minnesota Studies in the Philosophy of Science.

${ }^{14}$ Consider a possible world inhabited by 'epiphenomenal' consciousnesses able to experience events at their locations but not capable of exerting causal influence. Now imagine causal signals to be confined to a subclass of paths in this world, as in ours, but these consciousnesses able to traverse what are spacelike paths. Would not the 'epistemic basis' for our spacetime topology in such a world outrun the continuity of causal paths in it that it would include the directly experiencible continuity of the paths traversable by a consciousness but not by a causal signal? Is such a world imaginable? Why not? On the other hand, one is, at least at first, more skeptical of the possibility of a world where the causal paths outrun those traversable by a consciousness, for just how would we 'ultimately' establish the genidentity of the signals marking out these causal paths not open to the direct topological inspection of some idealized local consciousness? Would, if such a world were possible, our epistemic basis for the topology once again be limited to the (now narrower) class of 'directly experiencible' paths? 